\title{
A High-Order Compact 2D FDFD Method for Waveguides
}

\author{
Gang Zheng' ${ }^{1}$ and Bing-Zhong Wang ${ }^{2}$ \\ ${ }^{1}$ State Key Laboratory of Satellite Ocean Environment Dynamics, Second Institute of Oceanography, State Oceanic Administration, \\ Hangzhou 310012, China \\ ${ }^{2}$ Institute of Applied Physics, University of Electronic Science and Technology of China, Chengdu 610054, China
}

Correspondence should be addressed to Gang Zheng, zhenguestc@gmail.com

Received 7 May 2012; Accepted 15 July 2012

Academic Editor: Zhongxiang Q. Shen

Copyright ( $) 2012$ G. Zheng and B.-Z. Wang. This is an open access article distributed under the Creative Commons Attribution License, which permits unrestricted use, distribution, and reproduction in any medium, provided the original work is properly cited.

\begin{abstract}
A high-order compact two-dimensional finite-difference frequency-domain (2D FDFD) method is proposed for the analysis of the dispersion characteristics of waveguides. A surface impedance boundary condition (SIBC) for the high-order compact 2D FDFD method is also given to model lossy metal waveguides. Four transverse field components are involved in the final eigenequation. Numerical examples are given, which show that this high-order compact 2D FDFD method is more efficient than the low-order compact 2D FDFD method and has a less storage cost.
\end{abstract}

\section{Introduction}

In practical engineering designs, it is very important to accurately and efficiently analyze the dispersion characteristics of waveguides. A two-dimensional finite-difference frequency-domain (2D FDFD) method was proposed for this purpose [1]. A compact 2D FDFD method was brought forward later in which only four transverse field components are involved in the final eigenequation [2]. In 2D FDFD methods, the cross-section of wave guides is discretized with compact Yee's meshes, and corresponding difference equations are set up on the nodes. A matrix eigenequation can be established from them finally. The dispersion characteristics can be obtained by solving this eigenequation. Unlike in two-dimensional finite-difference time-domain (2D FDTD) methods $[3,4]$, in 2D FDFD methods the complex propagation constant can be found at a given frequency directly and there is no need of the discrete Fourier transform. Another advantage of 2D FDFD methods is that the dispersion characteristics of several modes at a given frequency can be analyzed at the same time.

In a low-order finite-difference method, fine meshes need to be used in order to obtain a high accuracy, and this is very time consuming. However, under the same spatial discretization, the accuracy can be largely improved by a high order finite-difference method.

In $2 \mathrm{D}$ FDFD methods, the main computational time is spent on solving the final matrix eigenequation, and it increases as the size of the final matrix eigenequation increases. In this letter, a high-order compact 2D FDFD method is proposed for calculating complex propagation constants of waveguides, and a corresponding surface impedance boundary condition (SIBC) is also given to model lossy metal waveguides for the high-order compact $2 \mathrm{D}$ FDFD method. The numerical results show that under the same accuracy, the number of the meshes in the high order compact 2D FDFD method is much less than in the low-order compact 2D FDFD method. Correspondingly, both the computational time and the number of nonzero elements largely decrease in the former, and the burdens of computation and storage are reduced.

\section{Formulation}

2.1. The High-Order Compact 2D FDFD Method. Electric field $\mathbf{E}$ and magnetic field $\mathbf{H}$ are normalized with the square root of the free-space wave impedance $\left(\eta_{0}=\sqrt{\mu_{0} / \varepsilon_{0}}\right)$. It is 
assumed that waveguides are uniform in $z$-direction. Then the field of a mode in a waveguide can be expressed as

$$
\begin{aligned}
\mathbf{E}(x, y, z) & =\left[E_{x}(x, y) \mathbf{e}_{x}+E_{y}(x, y) \mathbf{e}_{y}+E_{z}(x, y) \mathbf{e}_{z}\right] e^{-\gamma z}, \\
\mathbf{H}(x, y, z) & =\left[H_{x}(x, y) \mathbf{e}_{x}+H_{y}(x, y) \mathbf{e}_{y}+H_{z}(x, y) \mathbf{e}_{z}\right] e^{-\gamma z},
\end{aligned}
$$

where $\gamma$ is the complex propagation constant of the mode. After substituting (1) into Maxwell's curl equations, the following equations are obtained:

$$
\begin{gathered}
-\frac{\partial E_{z}}{\partial x}+j k_{0} \mu_{r} H_{y}=\gamma E_{x}, \\
-\frac{\partial E_{z}}{\partial y}-j k_{0} \mu_{r} H_{x}=\gamma E_{y}, \\
-\frac{\partial H_{z}}{\partial x}-j k_{0} \varepsilon_{r} E_{y}=\gamma H_{x} \\
-\frac{\partial H_{z}}{\partial y}+j k_{0} \varepsilon_{r} E_{x}=\gamma H_{y} \\
\frac{\partial H_{x}}{\partial y}-\frac{\partial H_{y}}{\partial x}+j k_{0} \varepsilon_{r} E_{z}=0 \\
\frac{\partial E_{y}}{\partial x}-\frac{\partial E_{x}}{\partial y}+j k_{0} \mu_{r} H_{z}=0 .
\end{gathered}
$$

The cross-section of the waveguide is discretized with compact Yee's meshes (see Figure 1). After the forthorder central difference is used to approximate the partial derivatives in (2)-(7), the following difference equations are obtained:

$$
\begin{aligned}
\frac{1}{24 \Delta x} & {\left[E_{z}(i+2, j)-E_{z}(i-1, j)\right] } \\
& -\frac{9}{8 \Delta x}\left[E_{z}(i+1, j)-E_{z}(i, j)\right] \\
& +j k_{0} \mu_{r} H_{y}(i, j-1)=\gamma E_{x}(i, j), \\
\frac{1}{24 \Delta y}\left[E_{z}(i, j+2)-E_{z}(i, j-1)\right] & -\frac{9}{8 \Delta y}\left[E_{z}(i, j+1)-E_{z}(i, j)\right] \\
- & j k_{0} \mu_{r} H_{x}(i-1, j)=\gamma E_{y}(i, j), \\
\frac{1}{24 \Delta x} & {\left[H_{z}(i+2, j)-H_{z}(i-1, j)\right] } \\
& -\frac{9}{8 \Delta x}\left[H_{z}(i+1, j)-H_{z}(i, j)\right] \\
- & j k_{0} \varepsilon_{r} E_{y}(i+1, j)=\gamma H_{x}(i, j),
\end{aligned}
$$

$$
\begin{aligned}
& \frac{1}{24 \Delta y}\left[H_{z}(i, j+2)-H_{z}(i, j-1)\right] \\
& \quad-\frac{9}{8 \Delta y}\left[H_{z}(i, j+1)-H_{z}(i, j)\right] \\
& \quad+j k_{0} \varepsilon_{r} E_{x}(i, j+1)=\gamma H_{y}(i, j), \\
& \frac{1}{24 \Delta y}\left[H_{x}(i-1, j+1)-H_{x}(i-1, j-2)\right] \\
& \quad-\frac{9}{8 \Delta y}\left[H_{x}(i-1, j)-H_{x}(i-1, j-1)\right] \\
& \quad-\frac{1}{24 \Delta x}\left[H_{y}(i+1, j-1)-H_{y}(i-2, j-1)\right] \\
& \quad+\frac{9}{8 \Delta x}\left[H_{y}(i, j-1)-H_{y}(i-1, j-1)\right] \\
& \quad-j k_{0} \varepsilon_{r} E_{z}(i, j)=0, \\
& \frac{1}{24 \Delta x}
\end{aligned}
$$

where $\Delta x$ and $\Delta y$ are mesh sizes in $x$ - and $y$-directions, respectively. These high-order difference equations are set up on all corresponding nodes except the nodes near the interface between two materials. Mixed-order difference equations are set up on these nodes, in which the secondorder central difference approximation is used for the normal derivative and the forth-order central difference approximation is used for the tangential derivative.

2.2. The SIBC for the High-Order Compact 2D FDFD Method. For lossy metal, the tangential electric field $\mathbf{E}_{\text {tan }}$ and the tangential magnetic field $\mathbf{H}_{\text {tan }}$ on its surface satisfy

$$
\mathbf{E}_{\mathrm{tan}}=Z_{s}\left(\mathbf{n} \times \mathbf{H}_{\tan }\right),
$$

where $\mathbf{n}$ is a unit vector normal to the surface of lossy metal (see Figure 2). The normalized surface impedance $Z_{s}$ is

$$
Z_{s}=\frac{(1+j)}{\left(\sigma \delta \eta_{0}\right)}
$$

where $\sigma$ and $\delta$ are the conductivity and the skin depth of lossy metal, respectively.

The surface in Figure 2(a) is considered here, and the other surfaces of lossy metal can be treated in the same way. 


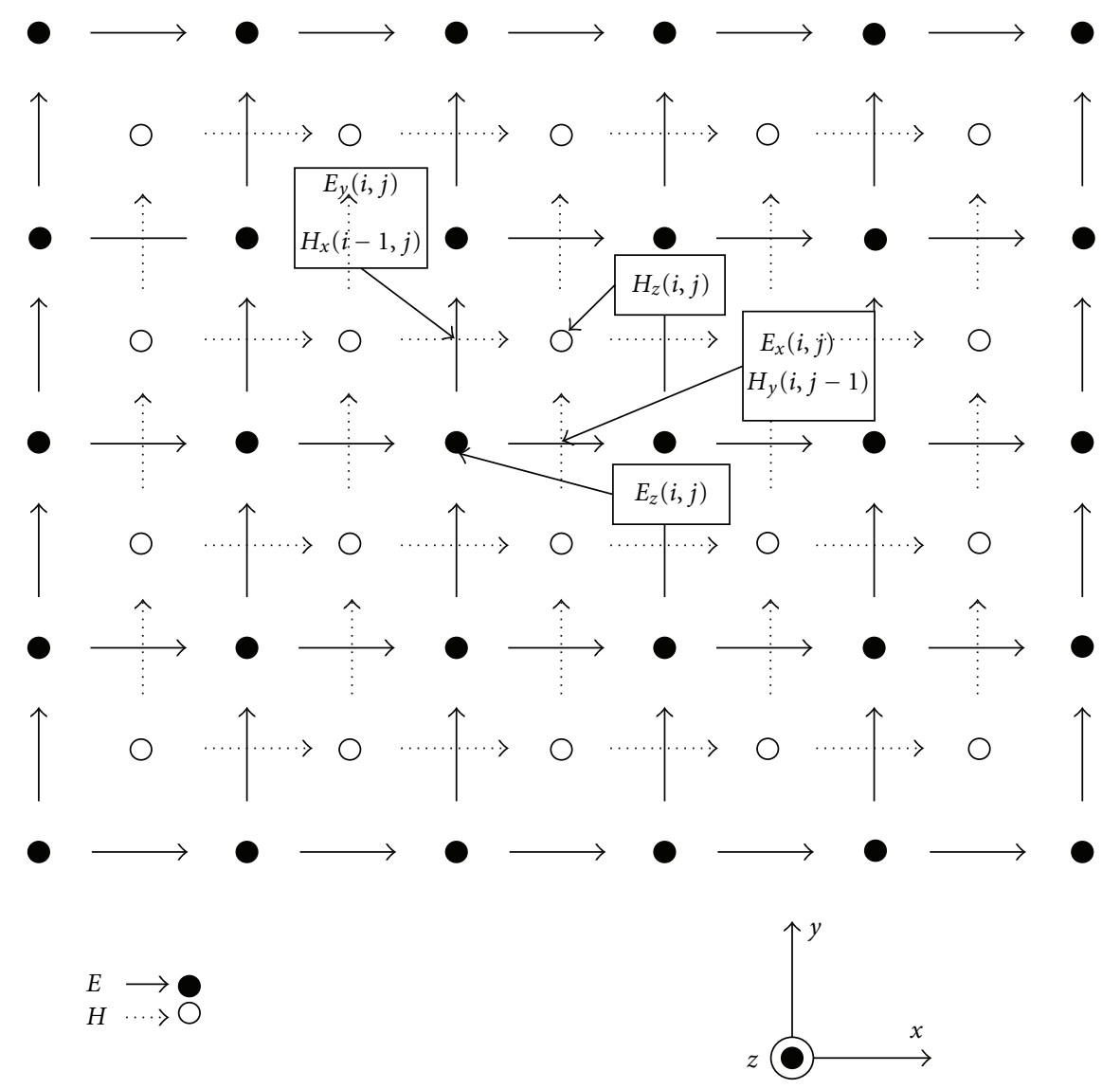

Figure 1: Compact Yee's meshes.

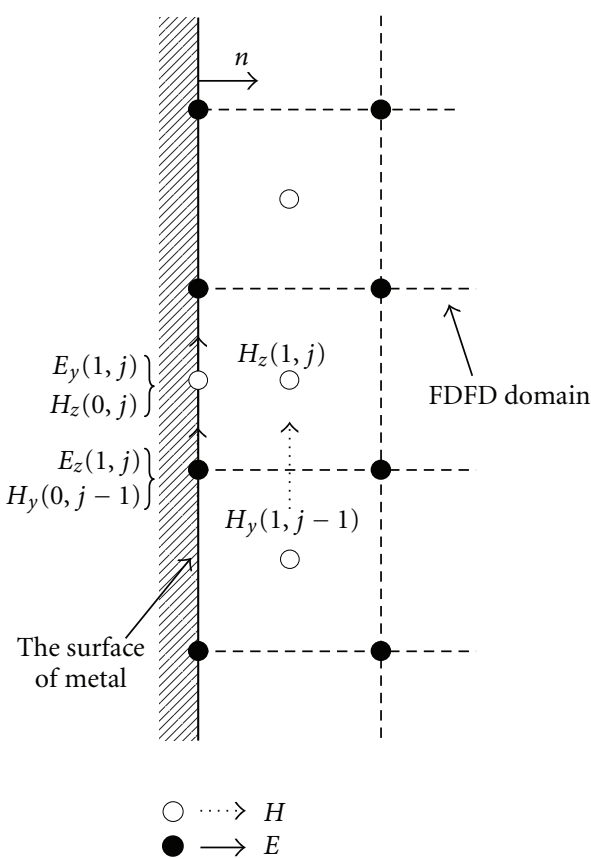

(a)

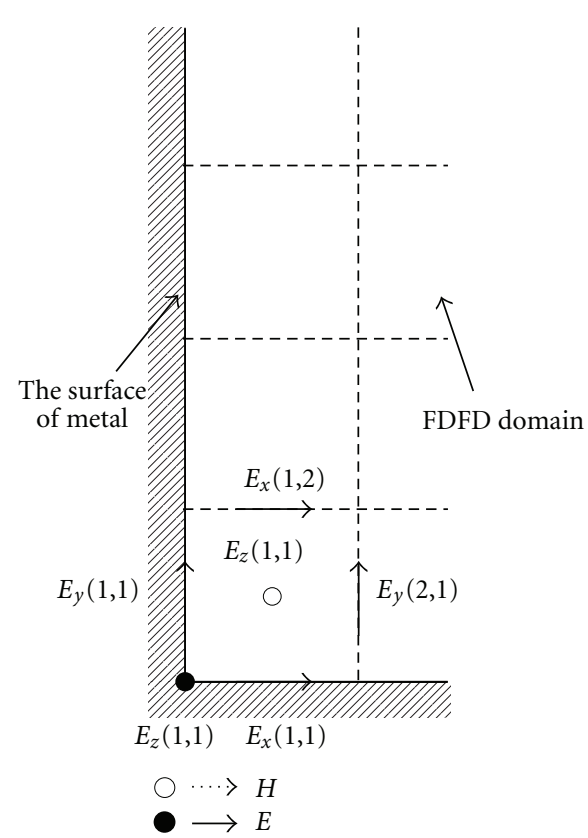

(b)

Figure 2: (a) A surface of lossy metal. (b) A corner of lossy metal. 
It can be seen that the $\mathbf{E}_{\mathrm{tan}}$ nodes of meshes coincide with the surface of lossy metal, but the $\mathbf{H}_{\tan }$ nodes of meshes do not coincide with the surface. Therefore, the following approximation is taken

$$
\begin{aligned}
& H_{y}(0, j) \approx H_{y}(1, j), \\
& H_{z}(0, j) \approx H_{z}(1, j) .
\end{aligned}
$$

The discrete form of (14) on the surface is

$$
\begin{gathered}
E_{y}(1, j)=-Z_{s} H_{z}(0, j), \\
E_{z}(1, j)=Z_{s} H_{y}(0, j-1) .
\end{gathered}
$$

After substituting (16) into (19), the SIBC for $E_{z}$ on the surface is obtained as follows

$$
E_{z}(1, j)=Z_{s} H_{y}(1, j-1) .
$$

Now the SIBC for the remaining tangential component of the electric field on the surface is considered. After substituting (17) into (18), we have

$$
E_{y}(1, j)=-Z_{s} H_{z}(1, j)
$$

The mixed-order difference equation is set up on the node $H_{z}(1, j)$ as

$$
\begin{aligned}
& \frac{1}{\Delta x}\left[E_{y}(2, j)-E_{y}(1, j)\right] \\
& \quad+\frac{1}{24 \Delta y}\left[E_{x}(1, j+2)-E_{x}(1, j-1)\right] \\
& \quad-\frac{9}{8 \Delta y}\left[E_{x}(1, j+1)-E_{x}(1, j)\right] \\
& +j k_{0} \mu_{r} H_{z}(1, j)=0 .
\end{aligned}
$$

Substituting (22) into (21) to eliminate $H_{z}(1, j)$, then the SIBC for $E_{y}$ on the surface is obtained as

$$
\begin{aligned}
E_{y}(1, j)= & \frac{Z_{s} \Delta x}{j k_{0} \mu_{r} \Delta x+Z_{s}} \\
& \times\left\{\begin{array}{c}
\frac{E_{y}(2, j)}{\Delta x}-\frac{9}{8 \Delta y}\left[E_{x}(1, j+1)-E_{x}(1, j)\right] \\
+\frac{1}{24 \Delta y}\left[E_{x}(1, j+2)-E_{x}(1, j-1)\right]
\end{array}\right\} .
\end{aligned}
$$

Now the SIBC for $E_{x}(1,1)$ and $E_{y}(1,1)$ in the corner (see Figure 2(b)) is considered. They can be expressed as

$$
\begin{gathered}
E_{y}(1,1)=-Z_{s} H_{z}(1,1) \\
E_{x}(1,1)=Z_{s} H_{z}(1,1) .
\end{gathered}
$$

After combining (24) with the low-order difference equation on the node $H_{z}(1,1)$, the SIBC in this corner is obtained

$$
\begin{aligned}
E_{x}(1,1)= & \frac{Z_{s}}{j k_{0} \mu_{r} \Delta x \Delta y+Z_{s}(\Delta x+\Delta y)} \\
& \times\left[\Delta x E_{x}(1,2)-\Delta y E_{y}(2,1)\right] \\
E_{y}(1,1)= & \frac{Z_{s}}{j k_{0} \mu_{r} \Delta x \Delta y+Z_{s}(\Delta x+\Delta y)} \\
& \times\left[\Delta y E_{y}(2,1)-\Delta x E_{x}(1,2)\right] .
\end{aligned}
$$

2.3. The Final Eigenequation. From (8) to (13) and the SIBC, (26) is obtained as

$$
\left[\begin{array}{ccccccc}
0 & A_{1,2} & A_{1,3} & 0 & 0 & 0 & A_{1,7} \\
A_{2,1} & 0 & 0 & A_{2,4} & 0 & 0 & 0 \\
0 & A_{3,2} & A_{3,3} & 0 & 0 & 0 & 0 \\
A_{4,1} & 0 & 0 & A_{4,4} & A_{4,5} & A_{4,6} & 0 \\
A_{5,1} & 0 & 0 & 0 & A_{5,5} & A_{5,6} & 0 \\
A_{6,1} & 0 & 0 & 0 & 0 & A_{6,6} & 0 \\
0 & A_{7,2} & 0 & 0 & 0 & 0 & A_{7,7}
\end{array}\right]\left[\begin{array}{l}
x_{1} \\
x_{2} \\
x_{3} \\
x_{4} \\
x_{5} \\
x_{6} \\
x_{7}
\end{array}\right]=\gamma\left[\begin{array}{c}
x_{1} \\
x_{2} \\
0 \\
0 \\
0 \\
0 \\
0
\end{array}\right],
$$

where $x_{1}, x_{2}, x_{3}, x_{4}, x_{5}, x_{6}$, and $x_{7}$ are all column vectors. The vector $x_{1}$ represents the transverse electric field inside the FDFD domain, the vector $x_{2}$ represents the transverse magnetic field inside the FDFD domain, the vector $x_{3}$ represents the $z$-direction electric field inside the FDFD domain, the vector $x_{4}$ represents the $z$-direction magnetic field inside the FDFD domain, the vector $x_{5}$ represents the transverse tangential component of the electric field on the surface of lossy metal, the vector $x_{6}$ represents the transverse tangential component of the electric field in the corners, and the vector $x_{7}$ represents the $z$-direction electric field on the surface of lossy metal.

After eliminating $x_{3}, x_{4}, x_{5}, x_{6}$, and $x_{7}$ in (26), the following eigenequation is derived:

$$
\left[\begin{array}{cc}
0 & B_{1,2} \\
B_{2,1} & 0
\end{array}\right]\left[\begin{array}{l}
x_{1} \\
x_{2}
\end{array}\right]=\gamma\left[\begin{array}{l}
x_{1} \\
x_{2}
\end{array}\right],
$$

where

$$
\begin{gathered}
B_{1,2}=A_{1,2}-A_{1,3} A_{3,3}^{-1} A_{3,2}-A_{1,7} A_{7,7}^{-1} A_{7,2}, \\
B_{2,1}=A_{2,1}-A_{2,4} A_{4,4}^{-1}\left[\begin{array}{c}
A_{4,5} A_{5,5}^{-1}\left(A_{5,6} A_{6,6}^{-1} A_{6,1}-A_{5,1}\right) \\
-A_{4,6} A_{6,6}^{-1} A_{6,1}+A_{4,1}
\end{array}\right] .
\end{gathered}
$$

The matrix blocks $A_{3,3}, A_{4,4}, A_{5,5}, A_{6,6}$ and $A_{7,7}$ are full rank and have nonzero elements only on their diagonal lines. So their inverses are very easy to obtain and the matrix in (27) is still very sparse. After solving (27), the complex propagation constants of the modes in the waveguide can be obtained.

\section{Numerical Results}

The dispersion characteristics of a rectangular metal waveguide with the size of $19.05 \mathrm{~mm} \times 9 \mathrm{~mm}$ are respectively 


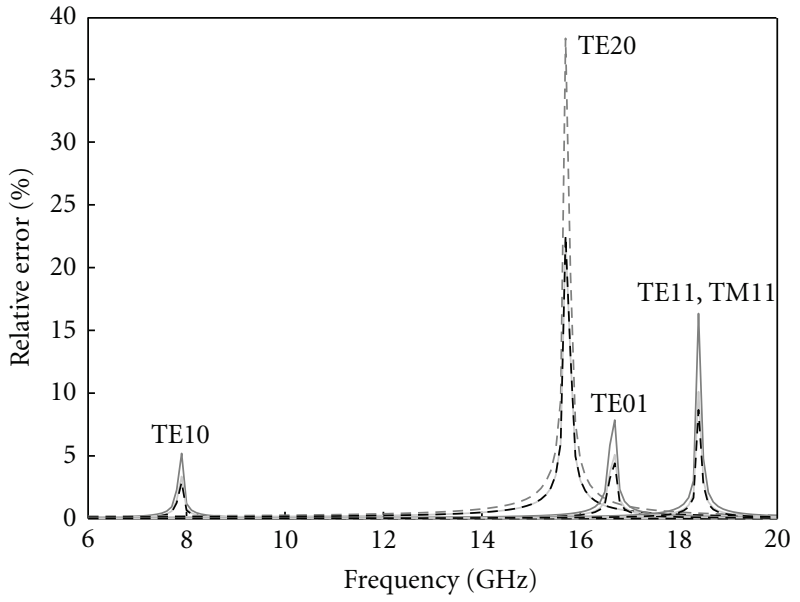

- The low-order compact 2D FDFD method with $32 \times 32$ meshes - The low-order compact 2D FDFD method with $40 \times 40$ meshes

— The high-order compact 2D FDFD method with $16 \times 16$ meshes

FIGURE 3: The relative errors of the complex propagation constants obtained by the two methods, respectively.

TABle 1: The computational times and the number of nonzero elements.

\begin{tabular}{lcc}
\hline Method & $\begin{array}{c}\text { Computational time } \\
(\mathrm{s})\end{array}$ & $\begin{array}{c}\text { Number of } \\
\text { nonzero elements }\end{array}$ \\
\hline $\begin{array}{l}\text { Low order }(32 \times 32 \\
\text { meshes })\end{array}$ & 166 & 27028 \\
$\begin{array}{l}\text { Low order }(40 \times 40 \\
\text { meshes })\end{array}$ & 418 & 42740 \\
$\begin{array}{l}\text { High order }(16 \times 16 \\
\text { meshes })\end{array}$ & 28 & 18336
\end{tabular}

analyzed by the low-order compact 2D FDFD method and the high order compact 2D FDFD method, in order to compare their computational times and the numbers of nonzero elements of the matrices in the final eigenequations. Both of the two methods are programmed with Matlab 7.0 and running in a P4 desktop computer (CPU: $3.2 \mathrm{GHz}$ ).

Firstly, the metal is assumed to be lossless. The complex propagation constant of the TE10 mode is calculated at 141 frequency points by the low-order compact 2D FDFD method with $40 \times 40$ compact Yee's meshes in the crosssection, the low-order compact 2D FDFD method with $32 \times 32$ compact Yee's meshes and the high order compact $2 \mathrm{D}$ FDFD method with $16 \times 16$ compact Yee's meshes, respectively. The analytical formula of the TE10 mode is used as a standard and the relative errors of the two methods are shown in Figure 3. The curve of the high order compact 2D FDFD method with the coarsest meshes is in a very good agreement with the curve of the low-order compact 2D FDFD method with the finest meshes. The total computational times of solving the final eigenequations at all frequency sample points and the numbers of nonzero elements of the matrices in the final eigenequations are shown in Table 1 for the two methods, respectively. It can be

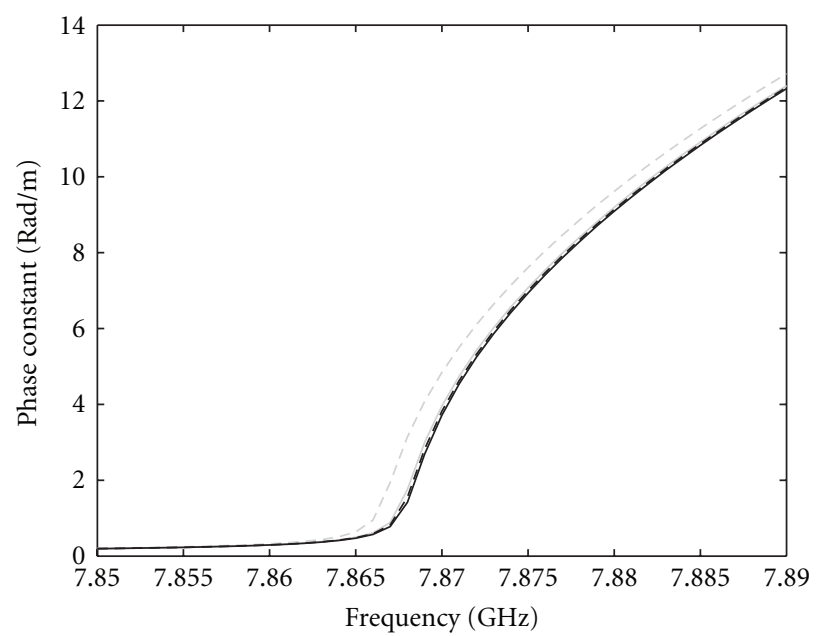

- The low-order compact 2D FDFD method with $32 \times 32$ meshes The low-order compact 2D FDFD method with $40 \times 40$ meshes — The high-order compact 2D FDFD method with $16 \times 16$ meshes -- - HFSS

(a)

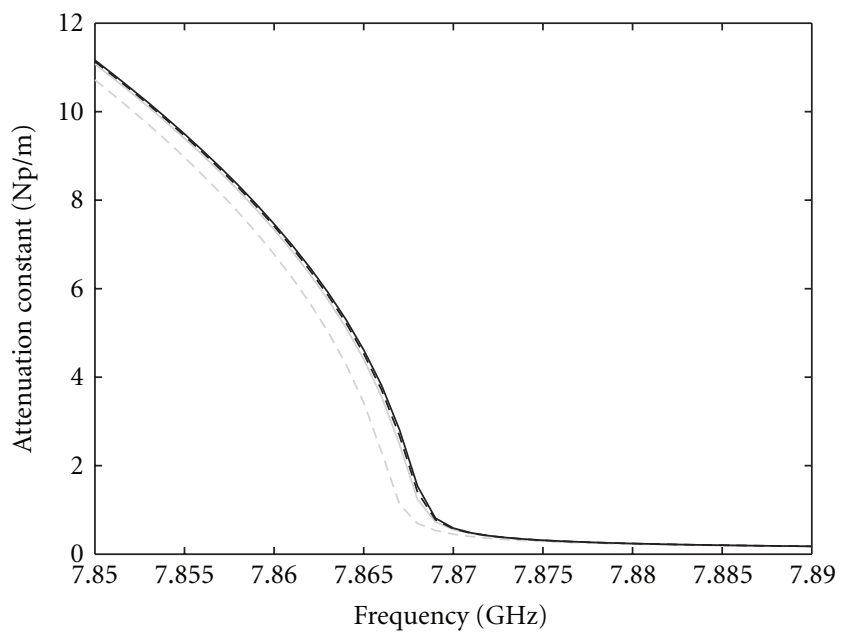

The low-order compact 2D FDFD method with $32 \times 32$ meshes
The low-order compact 2D FDFD method with $40 \times 40$ meshes
- The high-order compact 2D FDFD method with $16 \times 16$ meshes
-- HFSS

(b)

Figure 4: (a) The phase constant of mode 1. (b) The attenuation constant of mode 1 .

seen that both of them are the least in the high order compact 2D FDFD method without loss of accuracy. And these mean high computational efficiency and low storage cost. The complex propagation constants of other modes are also calculated, and the relative errors are shown in Figure 3, too.

Secondly, the metal is assumed with a conductivity $\sigma=$ $5.8 \times 10^{7} \mathrm{~S} / \mathrm{m}$. The complex propagation constants of mode 1 and mode 4 are calculated by the two methods, which are, respectively, corresponding to the TE10 mode and the TM11 mode of the lossless metal waveguide. The results of the two methods are compared with the results of Ansoft-HFSS, and they are shown in Figures 4 and 5 from the cutoff areas 


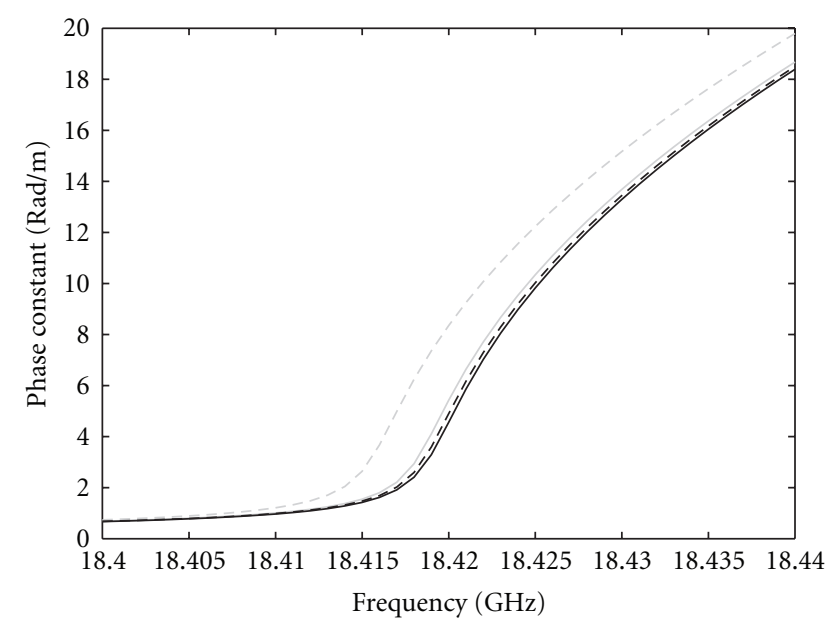

- . The low-order compact 2D FDFD method with $32 \times 32$ meshes The low-order compact 2D FDFD method with $40 \times 40$ meshes

- The high-order compact 2D FDFD method with $16 \times 16$ meshes - - - HFSS

(a)

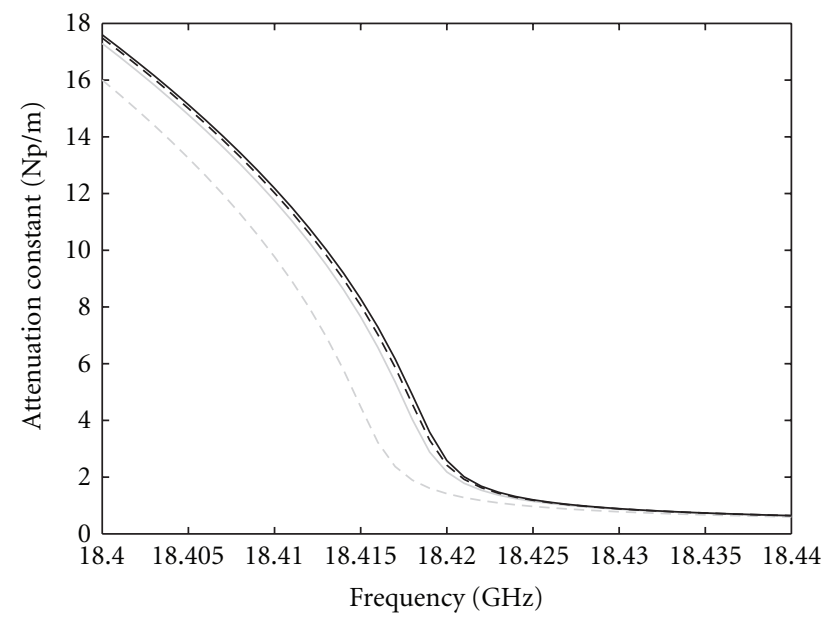

- - The low-order compact 2D FDFD method with $32 \times 32$ meshes The low-order compact 2D FDFD method with $40 \times 40$ meshes - The high-order compact 2D FDFD method with $16 \times 16$ meshes - - - HFSS

(b)

Figure 5: (a) The phase constant of mode 4. (b) The attenuation constant of mode 4 .

to the propagation areas. It can be seen that although the coarsest meshes are used in the high order compact 2D FDFD method, the results of it and Ansoft-HFSS are still in a very good agreement in both cases of mode 1 and mode 4 . However, the curves of the low-order compact 2D FDFD method with coarser meshes are not consistent with the others, but its curves tend to the others as the meshes become finer.

\section{Conclusion}

In this letter, a high-order compact two-dimensional finite-difference frequency-domain (2D FDFD) method is proposed for analyzing the dispersion characteristics of waveguides. A corresponding surface impedance boundary condition (SIBC) is given to model lossy metal waveguides. Some low-order approximations are used in the SIBC. However, the high order compact 2D FDFD method with the SIBC should be more accurate than the low-order compact 2D FDFD method, because the forth-order central difference is used in the most part of computational domain. In the numerical examples, the high order compact 2D FDFD method is compared with the low-order compact 2D FDFD method, and both of the cases of lossless and lossy metal are tested. The results show that both phase constants and attenuation constants can be accurately calculated through the high order compact 2D FDFD method, but both of the computational time and the storage cost are largely reduced.

\section{Acknowledgment}

This work was supported by the Doctoral Program of Higher Education of China (no. 20060614005).

\section{References}

[1] M. L. Lui and Z. Chen, "A direct computation of propagation constant using compact 2-D full-wave eigen-based finitedifference frequency-domain technique," in Proceedings of the International Conference on Computational Electromagnetics and Its Applications (ICCEA'99), pp. 78-81, Beijing, China, November 1999.

[2] Y.-J. Zhao, K.-L. Wu, and K.-K. M. Cheng, "A compact 2-D fullwave finite-difference frequency-domain method for general guided wave structures," IEEE Transactions on Microwave Theory and Techniques, vol. 50, no. 7, pp. 1844-1848, 2002.

[3] S. Xiao, R. Vahldieck, and H. Jin, "Full-wave analysis of guided wave structures using a novel 2-D FDTD," IEEE Microwave and Guided Wave Letters, vol. 2, no. 5, pp. 165-167, 1992.

[4] A. Asi and L. Shafai, "Dispersion analysis of anisotropic inhomogeneous waveguides using compact 2D-FDTD," Electronics Letters, vol. 28, no. 15, pp. 1451-1452, 1992. 

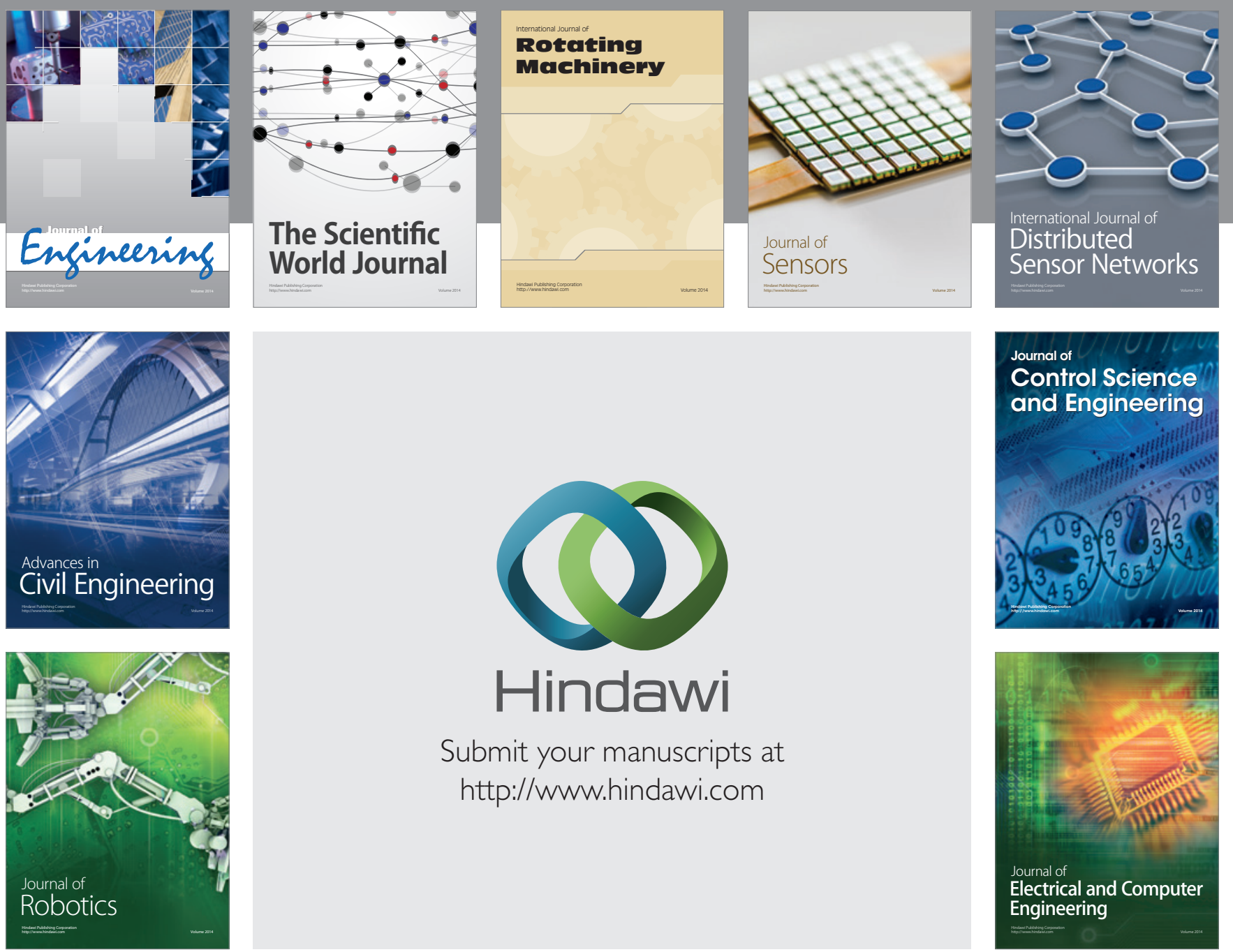

Submit your manuscripts at

http://www.hindawi.com
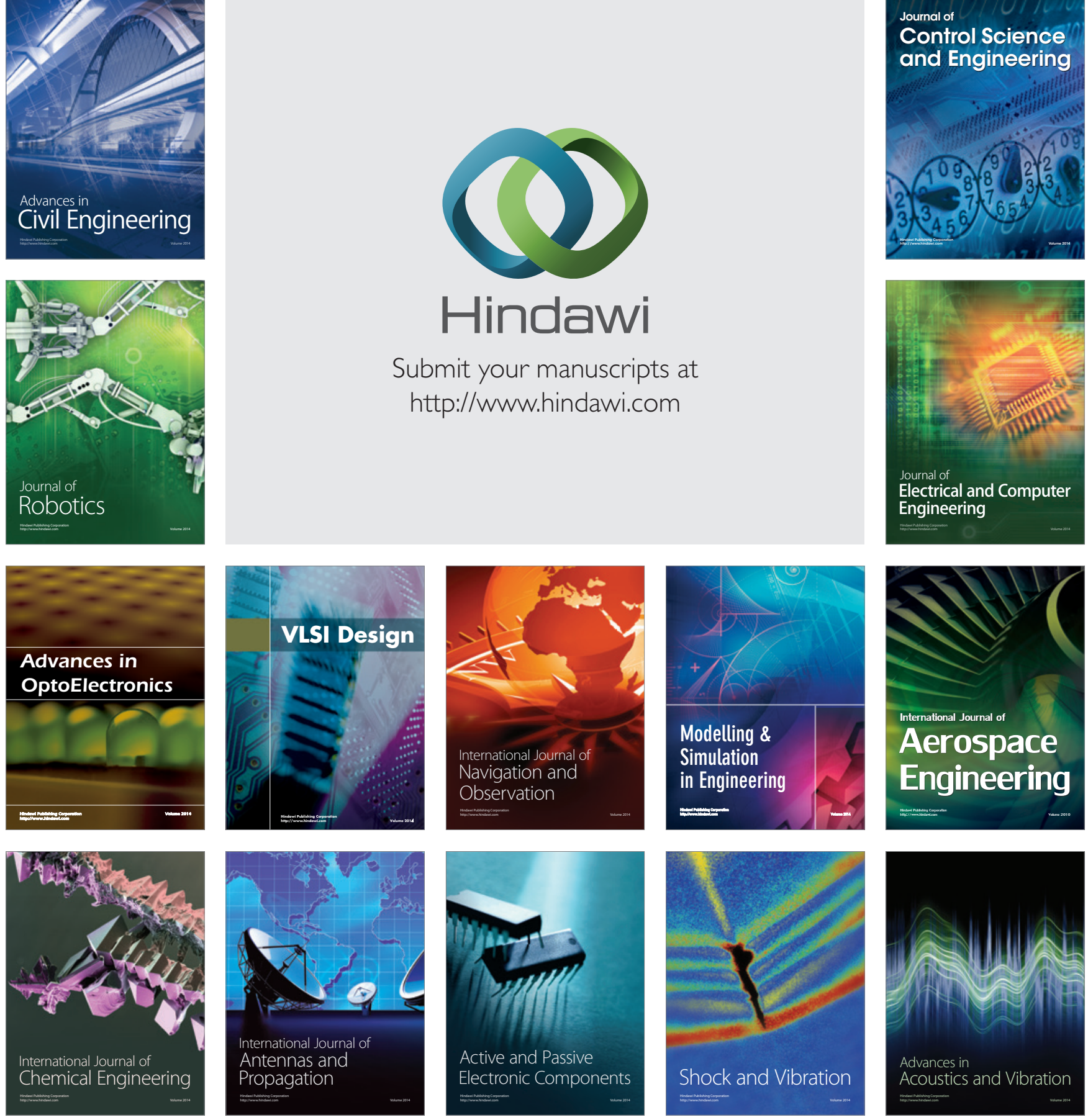